\title{
TRANSIÇÃO ALIMENTAR EM RECÉM-NASCIDOS COM DISPLASIA BRONCOPULMONAR
}

\author{
Transition time for full oral feeding in newborns \\ with bronchopulmonary dysplasia
}

\author{
Daiana Evangelista ${ }^{(1)}$, Andressa Oliveira ${ }^{(2)}$
}

\begin{abstract}
RESUMO
Objetivo: verificar o tempo de transição da sonda para a via oral plena nos lactentes com Displasia Broncopulmonar (DBP) e descrever as intercorrências observadas durante os períodos de alimentação. Métodos: revisão de prontuários do setor de Fonoaudiologia de 32 lactentes nascidos no Hospital Universitário Pedro Ernesto (HUPE/UERJ) divididos em 2 grupos. Grupo 1: 18 lactentes com diagnóstico de DBP; grupo 2: 18 lactentes com idade gestacional entre 29 e 32 semanas sem complicações clínicas relevantes que foram utilizados como grupo controle. Foram registrados o tempo de transição alimentar, as intercorrências durante a alimentação e via oral na alta hospitalar de ambos os grupos. Resultados: média e desvio padrão do tempo de transição alimentar dos grupos 1 e 2 respectivamente: 18,22 dias e 14,79; 6,50 dias e 3,68, com $p=0,002$. Nos broncodisplásicos foram comuns intercorrências respiratórias, dificuldade de coordenação sucçãoXrespiraçãoXdeglutição, dificuldades no padrão oral, além de sinais de retraimento e rebaixamento do estado de consciência durante a alimentação. Apesar disso, 10 lactentes (52,63\%) tiveram alta em aleitamento materno exclusivo e $3(15,78 \%)$ em aleitamento misto. Conclusão: pacientes com DBP precisaram de um período maior de treino de VO (18 dias), além de serem passíveis de intercorrências durante a alimentação. No entanto, constata-se que foi possível o aleitamento materno exclusivo.
\end{abstract}

DESCRITORES: Displasia Broncopulmonar; Recém-Nascidos; Alimentação

\section{INTRODUÇÃO}

Nos EUA, anualmente, 3.000 a 7.000 neonatos são considerados broncodisplásicos. De grande importância clínica, a displasia broncopulmonar (DBP) é também uma questão de saúde pública ${ }^{1}$. 0 aumento da incidência de doenças pulmonares crônicas, tais como a sibilância recorrente, associada ou não a refluxo gastresofágico, e a DBP, que é a complicação clínica mais severa; estão diretamente relacionadas com a sobrevivência de neonatos

(1) Fonoaudióloga; Coordenadora da equipe de Fonoaudiologia do Núcleo Perinatal/ Hospital Universitário Pedro Ernesto da Universidade do Estado do Rio de Janeiro, HUPE-UERJ, Rio de Janeiro, RJ; Especialista em Motricidade Orofacial.

(2) Fonoaudióloga; Treinamento Profissional Bolsista do setor de Fonoaudiologia do Hospital Universitário Pedro Ernesto da Universidade do Estado do Rio de Janeiro, HUPEUERJ, Rio de Janeiro, RJ; Especializanda em Motricidade Orofacial pelo CEFAC - Saúde e Educação. cada vez mais prematuros ${ }^{2-6}$. Atualmente, a DBP tem sido reconhecida como uma das principais causas de problemas respiratórios crônicos na infância, acarretando em dificuldades na alimentação, nutricionais e/ou no desenvolvimento neuropsicomotor $5,7,8$, este último devido à necessidade de internações prolongadas e/ou frequentes ${ }^{9}$.

A DBP é uma doença pulmonar crônica de etiologia multifatorial, influenciada pelo baixo peso de nascimento; infecção; oxigenoterapia suplementar e ventilação mecânica por tempo prolongado; edema pulmonar e prematuridade; entre outras. É pouco comum em neonatos com idade gestacional (IG) maior que 34 semanas e apresenta sinais radiológicos e de aumento de oxigênio inspirado ${ }^{1}$.

O tratamento é composto de medicamentos específicos tais como: broncodilatadores, diuréticos e corticosteróides 1,2,5,6; além de assistência respiratória e nutrição adequadas ${ }^{1,5}$. A questão nutricional é muito valorizada, pois os recém-nascidos com DBP apresentam um trabalho respiratório 
aumentado, o que faz com que estes tenham maior gasto energético $1,2,4,5,7,10-12$.

É real que a evolução clínica do paciente com DBP deve-se à intervenção de uma equipe multidisciplinar; desde o neonatologista, além de fisioterapeuta, o nutricionista e o fonoaudiólogo, entre outros de acordo com o quadro clínico de cada criança ${ }^{8}$. Apesar disso e das inúmeras pesquisas referentes à DBP nos últimos 20 anos, a grande maioria dos estudos no mundo concentram-se em padronizar critérios de diagnóstico, definir conceitos e incidências da doença. Na literatura consultada, poucos foram os estudos que procuraram descrever as dificuldades esperadas na alimentação ${ }^{13,14}$ e investigar estratégias eficientes para lhe dar com elas.

A escassez de fontes que correlacionem a DBP com a alimentação por via oral (VO) no período neonatal e a grande dificuldade desses bebês durante o processo de transição da sonda para a VO com inúmeras intercorrências respiratórias nesta etapa justificam uma investigação das características comumente encontradas nessa população para que aperfeiçoemos a qualidade da assistência através de intervenções mais efetivas.

O objetivo deste estudo foi verificar o tempo de transição da sonda para a via oral plena nos lactentes com DBP e descrever as intercorrências observadas nestes bebês durante os períodos de alimentação.

\section{MÉTODOS}

Trata-se de um estudo retrospectivo através de revisão de prontuários do setor de Fonoaudiologia do Hospital Universitário Pedro Ernesto da Universidade do Estado do Rio de Janeiro (HUPE/UERJ) de 18 recém nascidos (RN) / lactentes nascidos na unidade neonatal do referido hospital, no período de agosto de 2002 a junho de 2005. Todos os sujeitos tiveram diagnóstico de DBP pela equipe de Neonatologia, considerando-se broncodisplásicos os RN/lactentes que apresentaram dependência de oxigênio por período maior ou igual a 28 dias associado a achados radiológicos (grupo 1). Foram excluídos os RNs que tinham idade gestacional ao nascimento (IG) maior que 32 semanas, que possuíam malformações orais, cerebrais ou cardíacas, e os que não tinham diagnóstico de DBP.

Para comparar o tempo de transição alimentar foram revistos prontuários de $18 \mathrm{RNs}$ com IG menor ou igual há 32 semanas (ou seja, prematuros extremos, semelhantemente ao esperado de média de IG do grupo com diagnóstico de DBP), também atendidos pela equipe de Fonoaudiologia (grupo 2). Foram excluídos deste grupo os RNs com diagnóstico de asfixia, síndromes, displasia broncopulmonar, disfunções cardíacas e neurológicas e com hemorragia intracraniana graus III e IV.

A equipe de Fonoaudiologia da Unidade Neonatal no período referido da pesquisa era composta de uma staff, uma residente e três especializandas, sendo utilizados protocolo de avaliação e de seguimento e toda a equipe previamente capacitada no preenchimento destes.

Foram registrados para a pesquisa os seguintes parâmetros de ambos os grupos: idade gestacional (IG); sexo; peso de nascimento (PN); tempo total de suporte ventilatório (TTSV); idade gestacional corrigida (IGC), peso e quadro respiratório no início da transição alimentar; além das intercorrências durante a alimentação e forma de via oral na alta hospitalar.

A idade gestacional (IG) ao nascimento de ambos os grupos foi considerada pelo Ballard ${ }^{15}$, visto que muitos RNs não possuíam idade gestacional estimada pela data da última menstruação (DUM). Da mesma forma a idade gestacional corrigida (IGC) também foi calculada segundo Ballard ${ }^{15}$.

Foi considerado para o tempo total de suporte respiratório (TTSR) o somatório de dias de tubo orotraqueal, Pressão Positiva em Vias Aéreas (CPAP), oxyhood e cateter perilabial de oxigênio $\left(\mathrm{O}_{2}\right.$ inalatório). Alguns $\mathrm{RN} /$ lactentes necessitaram de $\mathrm{O}_{2}$ inalatório somente nos horários das dietas (uso intermitente). Esse tipo de suporte também foi considerado no número total de dias.

Para tempo de transição alimentar (TTA) foi considerado o número de dias que o RN/ lactente necessitou para conseguir se alimentar exclusivamente por via oral, a partir do início do seu treino até a suspensão da sonda de alimentação. Portanto, contabilizou-se a partir do dia em que o bebê conseguiu receber um volume $>5 \mathrm{ml}$ por VO (copo, finger-feeding, translactação ou mamadeira) ou permaneceu em seio materno (SM) sugando de maneira nutritiva por $>5 \mathrm{~min}$, e conseguiu manter esse padrão ou aperfeiçoá-lo nas dietas seguintes ou dias subsequentes, ganhando peso, constatando-se que não mais necessitava da sonda. Não foi incluído o período de estimulação da sucção não-nutritiva (digital ou no SM), estimulação gustativa ou sensório-motora-oral (SMO) prévio ao início da alimentação por VO no cálculo do tempo de transição alimentar (TTA), nem os dias em que foram realizados apenas avaliações da sucção nutritiva, mesmo que em dias consecutivos.

$\mathrm{Na}$ referida unidade, inicia-se o treino de VO pela Fonoaudiologia quando há estabilidade clínica, liberação da equipe de Neonatolologia e sinais de maturidade e interesse na alimentação por parte do bebê. Neste período o bebê recebe parte da 
alimentação por VO e complementação através da sonda de alimentação. Para avaliação da VO e transição alimentar a equipe de fonoaudiologia optava sempre pelo SM como primeira escolha. Técnicas alternativas de alimentação por VO muitas vezes eram necessárias, sendo estas: o copinho, finger-feeding, a translactação, ou a mamadeira. O copo era avaliado como rotina da unidade, a pedido da equipe de neonatologia, na tentativa de viabilizar a retirada da sonda e complementação do SM. Portanto, todas as crianças foram avaliadas no copo. Nos casos em que as crianças apresentaram dificuldades no copo, era avaliada a técnica de finger-feeding pela equipe de fonoaudiologia. $O$ finger-feeding, do inglês alimentação pelo dedo ou sonda-dedo, consiste numa técnica de alimentação VO concomitante a estimulação oral. Nesta técnica, uma sonda no 06 é fixada ao dedo mínimo enluvado do terapeuta através de um fino esparadrapo. A sonda é conectada a um recipiente (seringa ou copo) com complemento (leite humano ordenhado preferencialmente) que deve ser posicionado abaixo da altura da boca do bebê, de maneira que o leite flua por sucção e não por gravidade ${ }^{16,17}$. A translactação (ou relactação) foi utilizada nos lactentes cujas mães estavam com reduzida capacidade de lactação no período de transição alimentar. Esta é uma técnica que estimula o aleitamento materno e o aumento da produção láctea, através de uma sonda de alimentação $n^{\circ} 06$ ou 4 presa junto à mãe, estando o orifício de saída na altura do mamilo. $\mathrm{Na}$ outra extremidade é acoplado um recipiente ou seringa onde fica armazenado o leite que flui a partir da sucção do bebê ao seio materno ${ }^{17,18}$. A mamadeira não é utilizada como rotina na unidade por se tratar de um hospital com o título de Hospital Amigo da Criança, no entanto, nos casos em que era utilizada com broncodisplásicos optava-se pelo modelo Miniform Lillo by Gerber ${ }^{\circledR}$ com capacidade para $50 \mathrm{ml}$ e bico longo e de látex conhecido como "chuquinha".
Quanto às intercorrências durante a alimentação, estas foram agrupadas em 5 grupos: respiratórias (queda de saturação percutânea de oxigênio, cianose, esforço respiratório, batimento de asa do nariz); de coordenação sucçãoXrespiraçãoXdeglutição (S/R/D) (ritmo lento de sucção, engasgos, acúmulo em cavidade oral, cansaço, escape extraoral, tosse); sinais de retraimentos (agitação, irritação, recusa alimentar, soluço, expressão facial de desconforto); padrão oral (ruído na sucção, excursionamento exagerado de mandíbula, padrão de amassamento, movimento incoordenado de língua) e estado de consciência (não despertar com pouco interesse na alimentação).

A presente pesquisa foi aprovada pelo Comitê de Ética em Pesquisa do Hospital Universitário Pedro Ernesto sob o número 1263, sendo considerado sem risco e sem necessidade de termo de consentimento livre e esclarecido.

A análise estatística foi realizada em conjunto com o Laboratório Médico de Pesquisas Avançadas (LAMPADA) da Faculdade de Ciências Médicas da UERJ e foram utilizadas medidas de tendência central dos grupos 1 e 2 e teste t de student para comparar os dois grupos, considerando $5 \%$ para significância estatística $(p<0,05)$.

\section{RESULTADOS}

O tempo de transição alimentar (TA) no grupo de lactentes com DBP (grupo 1=18,22 dias) foi maior que nos prematuros que não desenvolveram esta doença (grupo 2=6,5 dias) com diferença estatisticamente significante $(p<0,002)$ (Tabela 1). Na presente amostra, um maior peso para o início da VO não demonstrou ter correlação positiva com o tempo total necessário para a transição alimentar $(p=0,20)$.

No grupo com DBP houve crianças que chegaram a necessitar de mais de 31 dias de transição alimentar, enquanto no grupo sem DBP a grande

Tabela 1 - Medidas de tendência central das variáveis dos grupos 1 e 2

\begin{tabular}{|c|c|c|c|c|c|c|c|c|c|}
\hline \multirow{2}{*}{ Variáveis } & \multicolumn{3}{|c|}{ Grupo 1} & \multicolumn{6}{|c|}{ Grupo 2} \\
\hline & $\mathrm{n}$ & média & mediana & DP & $n$ & média & mediana & DP & \\
\hline$I G$ & 18 & 29,07 & 28,8 & 1,57 & 18 & 30,81667 & 30,8 & 1,16 & \\
\hline TTSV & 18 & 39,89 & 39 & 14,36 & 9 & 7 & 7,5 & 5 & \\
\hline$I G C$ & 18 & 36,1 & 36,35 & 1,84 & 16 & 33,27333 & 33,2 & 1,011694 & \\
\hline Peso & 18 & 1824,44 & 1805 & 293,34 & 15 & 1619 & 1625 & 205,02 & \\
\hline TTA & 18 & 18,22 & 15 & 14,79 & 18 & 6,5 & 5,5 & 3,68 & $\mathrm{p}=0,002^{*}$ \\
\hline
\end{tabular}

Legenda: IG: idade gestacional; TTSV: tempo total de suporte ventilatório; IGC: idade gestacional corrigida no início da transição alimentar; Peso: no início da transição alimentar; TTA: tempo total de transição alimentar.

${ }^{*}$ Correlação entre TTA entre o grupo 1 e o grupo $2: p=0,002$

Correlação entre TTA vs peso do grupo $1: p=0,20$ 
maioria (16 de 18) precisou de um período máximo de 10 dias. A distribuição do tempo de transição alimentar em dias dos grupos 1 e 2 e a frequência em que ocorreu encontra-se representada na Tabela 2.

No grupo com DBP foram comuns intercorrências durante as tentativas de alimentação oral tais como: respiratórias; de coordenação S/R/D; sinais de retraimentos; pouco interesse na alimentação e orais (Tabela 3 ).
As dificuldades respiratórias durante a alimentação e de coordenação S/R/D foram mais comuns com a mamadeira $(66,66 \%$ e $83,33 \%$ respectivamente) e com o copo (50\% e $83,33 \%$ respectivamente) (Tabela 3 ). No grupo 2 , sem DBP, as intercorrências foram raras e portanto, não relatadas.

O aleitamento materno exclusivo na alta hospitalar foi possível em um grande número de bebês com DBP $(\mathrm{N}=10,52,63 \%)$ (Tabela 4$)$.

Tabela 2 - Distribuição por frequência do tempo de transição alimentar em dias dos grupos 1 e 2

\begin{tabular}{ccccc}
\hline & \multicolumn{2}{c}{ Grupo 1 } & \multicolumn{2}{c}{ Grupo 2 } \\
\hline Tempo (dias) & $\begin{array}{c}\text { Frequência } \\
\mathbf{n}\end{array}$ & $\begin{array}{c}\text { Frequência } \\
\text { acumulada } \\
\mathbf{n}\end{array}$ & $\begin{array}{c}\text { Frequência } \\
\mathbf{n}\end{array}$ & $\begin{array}{c}\text { Frequência } \\
\text { acumulada } \\
\mathbf{n}\end{array}$ \\
\hline 0 a 10 & 7 & 7 & 16 & 16 \\
11 a 20 & 4 & 11 & 2 & 18 \\
21 a 30 & 2 & 13 & 0 & \\
31 a 40 & 4 & 17 & 0 & \\
41 a 50 & 1 & 18 & 0 & \\
\hline
\end{tabular}

Tabela 3 - Técnicas de alimentação utilizadas durante a internação e intercorrências observadas no grupo 1

\begin{tabular}{|c|c|c|c|c|c|c|c|c|c|c|c|}
\hline \multirow{2}{*}{\multicolumn{2}{|c|}{$\begin{array}{l}\text { Métodos usados na } \\
\text { transição alimentar }\end{array}$}} & \multicolumn{2}{|c|}{ Respiratórias } & \multicolumn{2}{|c|}{$\begin{array}{c}\text { Sinais de } \\
\text { Retraimento }\end{array}$} & \multicolumn{2}{|c|}{$\begin{array}{l}\text { Coordenação } \\
\text { S/R/D }\end{array}$} & \multicolumn{2}{|c|}{ Padrão Oral } & \multicolumn{2}{|c|}{$\begin{array}{c}\text { Estado de } \\
\text { Consciência }\end{array}$} \\
\hline & & $\mathbf{N}$ & $\%$ & $\mathbf{N}$ & $\%$ & $\mathbf{N}$ & $\%$ & $\mathbf{N}$ & $\%$ & $\mathbf{N}$ & $\%$ \\
\hline $\begin{array}{l}\text { Finger- } \\
\text { Feeding }\end{array}$ & 12 & 5 & 41,67 & 4 & 33,33 & 6 & 50 & 4 & 33,33 & 9 & 75 \\
\hline Copo & 18 & 9 & 50 & 6 & 33,33 & 15 & 83,33 & 1 & 5,56 & 9 & 50 \\
\hline Translactação & 6 & 2 & 33,33 & 2 & 33,33 & 2 & 33,33 & 0 & 0 & 4 & 66,66 \\
\hline SM & 16 & 5 & 31,25 & 2 & 12,5 & 6 & 37,5 & 4 & 25 & 12 & 75 \\
\hline Mamadeira & 6 & 4 & 66,67 & 2 & 33,33 & 5 & 83,33 & 1 & 16,67 & 5 & 83,33 \\
\hline
\end{tabular}

Legenda: SM: seio materno, N: número de RNs.

Tabela 4 - Forma de via oral na alta hospitalar

\begin{tabular}{ccccc}
\hline \multirow{2}{*}{ Vo na alta } & \multicolumn{2}{c}{ Grupo 1 } & \multicolumn{2}{c}{ Grupo 2 } \\
\cline { 2 - 5 } & $\mathbf{n}$ & $\%$ & $\mathbf{n}$ & $\%$ \\
\hline SME & 10 & 52,63 & 12 & 70,59 \\
Copo & 0 & 0 & 0 & 0 \\
SM + copo & 3 & 15,78 & 4 & 23,52 \\
Mamadeira & 5 & 31,57 & 1 & 5,89 \\
total & 18 & 100 & 17 & 100 \\
\hline
\end{tabular}

Legenda: SME: seio materno exclusivo, SM: seio materno, $\mathrm{n}$ : número de RNs.

\section{DISCUSSÃO}

O TTA no grupo de lactentes com DBP foi maior, conforme esperado ${ }^{18,19}$, apesar dos grupos serem semelhantes quanto a IG ao nascimento e do maior peso deste grupo no início da TA. Observa-se, portanto, que esta doença respiratória interferiu muito na alimentação destes bebês tornando-a lenta e trabalhosa. Estes achados estão de acordo com estudo recente que constatou menor frequência de sucção com pressão intra-oral fraca, resultando em menos 
deglutições e menor eficiência na alimentação revelada através da relação volume/minuto, além de dificuldade de coordenação S/R/D em lactentes com DBP de grau leve a severa, sendo a performance diretamente relacionada ao grau da doença ${ }^{14}$. Apesar do conhecimento de que o crescimento pulmonar minimiza as repercussões da doença ${ }^{1,2}$, na presente amostra o peso não demonstrou interferência na habilidade de alimentação. Em estudo anterior com prematuros sem DBP também não houve correlação entre o TTA e o peso ${ }^{20}$.

Além da necessidade de um período maior de treino de VO, no grupo com DBP foi comum a presença de intercorrências durante as tentativas de alimentação oral. Tais dificuldades justificam a intervenção fonoaudiológica por ser este o profissional habilitado em distúrbios da deglutição ${ }^{7,12}$. Esta etapa final da internação exige do profissional de saúde e da família, paciência, acompanhamento constante e conhecimento das suas especificidades.

As dificuldades encontradas na alimentação podem ser explicadas pelo fato dos pacientes com DBP apresentarem maiores dificuldades em manter a energia necessária durante a alimentação, pois estes têm como característica um esforço respiratório mesmo em ocasiões naturais, podendo se intensificar no momento de se alimentar, uma vez que desempenham um esforço adicional, ou seja, precisam sugar e deglutir ${ }^{1,6,20,21}$. O RN broncodisplásico apresenta um intervalo entre os movimentos inspiratórios curto, restando pouco tempo para a deglutição, dificultando com isso a coordenação $S / D / R$. Além disso, apresenta uma baixa reserva respiratória fazendo com que o período de apneia da deglutição, embora curto, repercuta intensamente neste paciente ${ }^{5,6,11,20,21}$. Em pesquisas anteriores, já foram constatados níveis mais altos de $\mathrm{PCO}_{2}$ em bebês com DBP severa, que poderiam ser responsáveis pelos efeitos na performance da sucção ${ }^{14}$ ou ainda, pode ser possível que os achados de dificuldades na sucção sejam um mecanismo desses bebês em tentar manter a respiração uma vez que esta é mais importante que a alimentação oral para as crianças com comprometimento respiratório ${ }^{14}$.

A manutenção dos índices de oxigênio arterial é um dos pontos mais importantes no tratamento, já que a hipoxemia influencia no ganho ponderal e no desenvolvimento cerebral 1,2,5,11,12, devendo ser mantidos estáveis os níveis de saturação durante a alimentação, o sono ou a vigília. Desta forma, sugere-se que seja oferecido um aumento no suporte respiratório visando reduzir o risco de quedas na SpO2 e minimizar a hipóxia induzida por depressão respiratória ${ }^{14}$.

No presente estudo foi possível observar que as dificuldades respiratórias durante a alimentação e de coordenação $S / R / D$ foram mais comuns com a mamadeira e com o copo, possivelmente devido à dificuldade desses bebês em lidar com volumes maiores ou fluxo mais rápido ${ }^{14,22}$. Este achado seria contrário ao senso comum de que quanto mais fácil fluir o leite, maior a facilidade e menor o esforço na alimentação destes bebês. O copo, apesar de comprovadamente seguro para RNs sem doenças respiratórias ${ }^{16,23-25}$ e preconizado pela OMS, não demonstrou essa segurança nos RNs com DBP, além de representar um risco para o agravo da saúde pulmonar destes, devido a possibilidades de engasgos ${ }^{16,22,25}$. Em contrapartida, no seio materno (31,2\% e $37,5 \%$ respectivamente) e na translactação (33,33\% e 33,33\% respectivamente) foram as situações de alimentação em que os lactentes ficaram mais estáveis e confortáveis com relação a estes dois parâmetros analisados (dificuldades respiratórias e incoordenação $S / R / D$ ) ${ }^{14}$, sendo menos frequente os sinais de retraimento no SM quando comparado com todas as outras formas de alimentação.

Apesar do copo ser avaliado como rotina da unidade, pode-se observar que grande parte das crianças tiveram dificuldades com essa técnica devido a dificuldade de coordenação S/R/D já esperadas nas crianças com DBP ${ }^{8,14}$, sendo o copo substituído pelo o finger-feeding para treino da sucção em 12 crianças.

$\mathrm{Na}$ ausência da mãe e, portanto, inviabilidade do seio materno e translactação, o finger-feeding demonstrou ter sido a melhor técnica para aperfeiçoar a coordenação S/D/R (50\%) e minimizar os agravos respiratórios $(41,66 \%)$ durante a alimentação, evidenciado pela menor porcentagem de intercorrências comparado à mamadeira e ao copo. Possivelmente um bico mais resistente que o de látex utilizado e de baixo fluxo, demonstre também resultados mais positivos ${ }^{14}$.

O finger-feeding permite o treino da sucção e de parâmetros orais importantes para seu desempenho eficiente tais como: canulamento e chicoteamento de língua, vedamento labial e pressão intra-oral através de pressão no centro da língua e leve movimento de retirada do dedo do terapeuta da cavidade oral do bebê, além de adequação da sensibilidade. O ritmo de sucção e a coordenação S/R/D também podem ser aperfeiçoados através de pausas induzidas para a respiração a partir de pequenos grupos de sucções e, facilitando ou dificultando o fluxo de leite pela sonda. Com o treino oral houve melhora significativa no padrão oral, proporcionando a alta em SME em grande parte das crianças.

Uma outra característica dos RNs com DBP descritas na literatura é a alteração dos padrões orais, mesmo que não haja associação com uma disfunção 
cerebral (Tabela 3) ${ }^{7,11-14}$. Além disso, pode ser esperado nesses pacientes refluxo gastroesofágico (RGE) e obstrução de vias aéreas superiores justificadas pela própria imaturidade e tempo de sonda prolongado, que agravam as dificuldades respiratórias e alimentares ${ }^{8}$. As quatro crianças que tiveram dificuldades orais caracterizadas por excursão mandibular ampla (3) e vedamento labial ineficiente (1) inclusive no finger-feeding, tinham como diagnóstico médico além da DBP: HIC (2); anemia, infecção e RGE (1) e tocotraumatismo e hiperbilirrubinemia (1). Uma justificativa possível para uma menor porcentagem de dificuldades orais nas outras formas de alimentação (copo e mamadeira) quando comparada ao finger-feeding é a dificuldade do terapeuta em observar parâmetros orais de sucção nestas técnicas, por ser o copo um mecanismo que não utiliza sucção e sim sorvidas e na mamadeira a percepção ser mais subjetiva do que no fingerfeeding em que o terapeuta encontra-se com o dedo na cavidade oral da criança e, portanto, tem maior capacidade de perceber disfunções.

Apesar da Fonoaudiologia ter como meta atingir a alimentação por VO em seio materno, visa-se inicialmente o desenvolvimento desses pacientes garantindo para isso a nutrição necessária e adequada, independentemente de que forma ela será oferecida. Na presente pesquisa foi possível observar que o aleitamento materno exclusivo na alta hospitalar foi possível em um grande número de bebês com DBP além dos 3 em alimentação mista (SM + copo) com possibilidade de evoluir para AME. É provável que os lactentes que mantiveram padrão oral adequado e seguro no copo fossem portadores d eum tipo leve de DBP. As cinco crianças que tiveram alta na mamadeira foram devido a hipogalactia (1), óbito materno (1), quadro clínico grave com HIC III, convulsão, RGE, asfixia e infecção (2), com pouca melhora no padrão oral mesmo com as técnicas facilitadoras de sucção durante a TA, e mãe sem interesse pela amamentação no seio (1).

Os RNs com complicações mais severas (tais como hemorragia intracraniana, anemia, asfixia) não foram excluídos do grupo 1 por ser de interesse dos pesquisadores em acompanhar se os bebês com doenças associadas diferenciavam dos RNs que não as apresentavam. As crianças com quadro clínico grave não apresentaram discrepância nas intercorrências comparadas com as demais, sendo possível alta em AME em uma das três crianças que tiveram diagnóstico médico de HIC grau III. Em outro estudo em que foram constatadas importan- tes dificuldades orais nos RNs com DBP, havia sido excluídos aqueles com anomalias ou sequelas neurológicas, confirmando os achados desse estudo que a DBP por si só já interfere no padrão oral da sucção ${ }^{14}$.

No presente trabalho os RNs foram considerados broncodisplásicos quando apresentaram dependência de $\mathrm{O}_{2}$ por período maior ou igual a 28 dias associado à achados radiológicos, já que esta era a classificação utilizada no período. Observase que a recuperação clínica da DBP precisa de um longo período, pois está vinculada a superação das dificuldades na respiração e na alimentação. O investimento na manutenção da lactação nesta população é valioso, devendo a equipe de saúde juntar esforços no acompanhamento dessa clientela durante toda sua internação no que diz respeito ao incentivo à ordenha precoce e aproximação do binômio mãe-bebê e se necessário através da translactação, ainda mais numa população em que o longo período de internação pode representar real baixa na lactação materna.

Este trabalho visou documentar a experiência do setor de Fonoaudiologia do Hospital Universitário Pedro Ernesto no atendimento a recém-nascidos e lactentes com displasia broncopulmonar. No entanto, faz-se necessários estudos prospectivos randomizados complementares com relação às técnicas de alimentação nesta população.

\section{CONCLUSÃO}

Pacientes com DBP precisaram de um período maior de treino de VO quando comparados a prematuros sem DBP, além de serem passíveis de intercorrências durante a alimentação como: dificuldades respiratórias, de coordenação $S / R / D$, no padrão oral, além de sinais de retraimento e rebaixamento do estado de consciência durante a alimentação. A evolução cautelosa e gradativa diretamente no seio materno mostrou ser viável e a melhor opção. Devido a hipogalactia comum pelo longo tempo de internação, foi necessário fazer uso da técnica de translactação (relactação) visando atingir este fim. Na presença de dificuldades orais e de incoordenação S/R/D o finger-feeding demonstrou ser um importante recurso, devendo o copinho ser evitado. Constata-se que é possível o aleitamento materno exclusivo o que representa menor custo para a família e melhor prognóstico de saúde geral para o bebê. 


\begin{abstract}
Purpose: to check the time of transition from the feeding tube to oral feeding in newborns with Bronchopulmonar Dysplasia (BPD); observe the difficulties occurred during the oral feeding. Methods: the speech pathology department reviewed thirty-two past cases of newborns born in the Pedro Ernesto Hospital of Rio de Janeiro State University. The first group was composed of eighteen newborns with BPD, while a second group was composed of eighteen healthy newborns born between 29 and 32 weeks used as a control group. We observed in both groups the following items: time it took for the newborns to transition from the feeding tube to the oral one, the difficulties presented during feeding periods, and finally the type of oral alimentation used by the discharge time. Results: the mean and standard deviation of the sample were 18.22 days and 14.79, respectively; for the control group it was 6.50 and 3.68 , respectively $(p=0.002)$. The group with BPD showed respiratory stress, lack of coordination between suctions, breathing and swallowing, and suction dysfunction. They also fell asleep during alimentation, showing extreme physical strain and defensive behaviors. Despite these results, 10 newborns (52.63\%) were discharged on exclusive breastfeeding and $3(15,78 \%)$ on mixed feeding (cupfeeding with breastfeeding). Conclusion: patients with BPD need extended time (18 days) to learn oral feeding, and moreover, they are susceptible to intercurrence during feeding. Nevertheless, it was possible to testify an exclusive breastfeeding.
\end{abstract}

KEYWORDS: Bronchopulmonary Dysplasia; Infant, Newborn; Feeding

\section{REFERÊNCIAS}

1. Monte LF, Silva Filho LV, Miyoshi MH, Rozov T. Displasia broncopulmonar. J Pediatr. 2005; 81:99-110.

2. Friederich $\mathrm{L}$, Corso $\mathrm{AL}$, Jones $\mathrm{MH}$. Prognóstico pulmonar em prematuros. J Pediatr. 2005; 81(Supl1): S79-S88.

3. Bancalari E, Claure N, Sosenko IR. Bronchopulmonary dysplasia: changes in pathogenesis, epidemiology and definition. Semin Neonatol. 2003; 8(1):63-71.

4. Cunha GS, Mezzacappa Filho F, Ribeiro JD. Fatores maternos e neonatais na incidência de displasia broncopulmonar em recém-nascidos de muito baixo peso. J Pediatr. 2003; 79(6):550-6.

5. Procianoy RS. Displasia broncopulmonar. J Pediatr. 1998; 74(Supl1):S95-S98.

6. Tapia JL, Agost D, Alegria A, Standen J, Escobar $M$, Grandi $C$, et al. Bronchopulmonary dysplasia: incidence, risk factors and resource utilization in a population of South American very low birth weight infants. J Pediatr. 2006; 82(1):15-20.

7. Parad RB. Displasia broncopulomonar/Doença pulmonar crônica. In: Cloherty JP, Elchenuvald EC, Stark AR. Manual de Neonatologia. Rio de Janeiro: Guanabara Koogan; 2005. p. 336-45.

8. Maia APJS, Fraga CSA, Figueira MR, Pereira MPP. Displasia broncopulmonar In: Lanzelotte V. Manual de atenção integral ao desenvolvimento e reabilitação - SOPERJ. Rio de Janeiro: Revinter; 2007. p. 65-71.
9. Silva Filho LVF. Doença pulmonar crônica neonatal. J Pediatr 1998; 74(4):265-74.

10. Kurzner SI, Garg M, Bautista DB, Bader D, Merritt RJ, Warburton D, et al. Growth failure in infants with bronchopulmonary dysplasia: nutrition and elevated resting metabolic expenditure. Pediatrics. 1988; 81(3):379-84.

11. Ferlin MLS, Rugolo LMSS. Displasia broncopulmonar. In: Costa HPF, Marba ST. O recém-nascido de muito baixo peso. São Paulo: Atheneu; 2004. p. 423-40.

12. Garzi RP, Cerruti VQ, Ferreira VJA. A importância da respiração no processo de alimentação do recém-nascido prematuro: relato de caso. Rev CEFAC. 2003; 5(1):63-67.

13. Hernandez AM. Atuação fonoaudiológica com o sistema estomatognático e a função de alimentação. In: Hernandez AM, organizador. Conhecimentos essenciais para atender bem o neonato. São José dos Campos: Pulso; 2003. p. 47-78.

14. Mizuno K, Nishida Y, Taki M, Hibino S, Murase $M$, Sakurai $M$, et al. Infants with bronchopulmonary dysplasia suckle with weak pressures to maintain breathing during feeding. Pediatrics. 2007; 120(4):1035-42.

15. Naufel HG. Avaliação Física e Classificação. In: Costa HPF, Marba ST. O recém-nascido de muito baixo peso. São Paulo: Atheneu; 2004. p. 61-72.

16. Rios IJA. Técnicas de sucção nutritiva para recém-nascido prematuro. In: Rios IJA, organizador. Conhecimentos essenciais para atender bem em 
fonoaudiologia hospitalar. São José dos Campos: Pulso; 2003. p. 83-8.

17. Xavier C. Trabalho fonoaudiológico com bebês durante a fase hospitalar. In: Limongi SCO, organizador. Paralisia cerebral: processo terapêutico em linguagem e cognição (pontos de vista e abrangência). São Paulo: Pró-Fono; 2000. p. 75-118.

18. Lima GMS. Métodos especiais de alimentação: copinho - relactação - translactação. In: Rego JD.

Aleitamento materno. São Paulo: Atheneu; 2001. p. 265-278.

19. Bühler KECB. Introdução da alimentação via oral com o uso do copinho em recém-nascidos prétermos: critérios fonoaudiológicos. [dissertação] São Paulo (SP): Universidade de São Paulo; 2003. 20. Craig CM, Lee DN, Freer YN, Laing IA. Modulations in breathing patterns during intermittent feeding in term infants and preterm infants with bronchopulmonary dysplasia. Dev Med Child Neurol. 1999; 41:616-24.
21. Couto DE, Nemr K. Análise da prática da técnica do copinho em hospitais amigos da criança nos estados do Rio de Janeiro e São Paulo. Rev CEFAC. 2005; 7(4):448-59.

22. Marinelli KA, Burke GS, Dodd VL. A comparison of the safety of cupfeedings and bottlefeedings in premature infants whose mothers intend to breastfeeding. J Perinatol. 2001; 21(6):350-5.

23. Dowling DA, Méier PP, DiFiore JM, Blatz M, Martin RJ. Cup-feeding for preterm infants: mechanics and safety. J Hum Lact. 2002; 18(1):13-20.

24. López CP, Chiari BM, Guedes ZCF, Goulart AL, Kopelman BI. A utilização copo na complementação do aleitamento materno considerações fonoaudiológicas. In: Hernandez AM. Conhecimentos essenciais para atender bem o neonato. São José dos Campos: Pulso; 2003. p. 107-11.

25. Oddy WH, Glenn K. Implementing the baby friendly hospital initiative: the role of finger feeding. Breastfeed Rev. 2003; 11(1):5-10.
RECEBIDO EM: 26/07/2008

ACEITO EM: 12/10/2008

Endereço para correspondência:

Daiana Evagenlista

Rua Marques de Abrantes 26, cobertura 02

Rio de Janeiro - RJ

CEP: 22230-061

E-mail: daiana.evangelista@gmail.com 